\title{
DE LA FORMACIÓN PERIODÍSTICA A LA REALIDAD DE LOS MEDIOS DE COMUNICACIÓN
}

\section{FROM JOURNALISTIC TRAINING TO THE REALITY OF MEDIA}

\section{DA FORMAÇÃO JORNALÍSTICA À REALIDADE DOS MEIOS DE COMUNICAÇÃO}

\section{Páginas Juan Simón Cancino Peña}

42-58 jscancinop@libertadores.edu.co

Recibido

15 de septiembre de 2015

Comunicador social-periodista, magíster en docencia. Fundación Universitaria Los Libertadores, Colombia.

\section{Aceptado}

2 de noviembre de 2015 
Resumen

El presente artículo da cuenta del contraste entre los resultados de una encuesta realizada a nueve periodistas con responsabilidades editoriales de igual número de medios de comunicación de la ciudad de Bogotá, a quienes se indagó por la relevancia de diecisiete competencias de formación de egresados de facultades de comunicación y periodismo, en relación con lo señalado por los syllabus de quince asignaturas de la Facultad de Comunicación y Periodismo de la Fundación Universitaria

Los Libertadores.

\section{Palabras clave}

Syllabus, competencias de formación, encuestas, periodistas, medios de comunicación 


\section{Abstract}

This article deals with the contrast in the results of a poll performed on nine editorial journalists from an equal number of media from Bogotá. The subjects were ask about the relevance of seven professional skills in the graduates from the faculties of communication and journalism, these skills being present in the syllabus of fifteen subjects taught at Communication and Journalism Faculty of Universidad los Libertadores.

\section{Key words}

Syllabus, Professional Skills, Polls, Journalists, Media

\section{Resumo}

O presente artigo dá conta do contraste entre os resultados dum questionário realizado a nove jornalistas com responsabilidades editoriais de igual número de meios de comunicação da cidade de Bogotá, a quem se indagó pela relevância de dezessete competências de formação de graduados de faculdades de comunicação e jornalismo, em relação com o assinalado pelos syllabus de quinze matérias da Faculdade de Comunicação e Jornalismo da Fundación Universitaria Los Libertadores.

\section{Palavras-chave}

Syllabus, competências de formação, questionários, jornalistas, meios de comunicação 


\section{Introducción}

Más allá de la discusión en torno a si el periodismo es una disciplina o una ciencia, discusión que antes de estar agotada apenas comienza, surge la cuestión respecto a qué conocimientos son necesarios para la práctica de un periodismo de calidad y qué responsabilidad asumen o están en la responsabilidad de asumir las escuelas o facultades de comunicación y periodismo. En ese orden de ideas, una de las preguntas más recurrentes que emerge luego de que un profesional ha recibido su título de universidad, interrogante del cual no están exentos los egresados de las facultades de periodismo y comunicación, es si la institución en la que recibió su grado lo ha formado de acuerdo con las necesidades de las organizaciones adonde llegará a ejercer la profesión para la cual se ha preparado, todo eso bajo el contexto de una educación entendida como el proceso formativo cuyo objetivo es atender las demandas del aparato productivo a toda escala.

Quizás Ramonet (1999) brinde un elemento central para comprender la dimensión del periodista contemporáneo cuando señala que:

En la actualidad, informar es esencialmente hacer asistir a un acontecimiento, es decir, mostrarlo, situarse a un nivel en el que el objetivo consiste en decir que la mejor manera de informarse equivale a informarse directamente. Es ésta la relación que pone en cuestión al propio periodismo. (p. 2).

De allí la importancia que tiene que los periodistas egresados de las escuelas de formación sean formados no sólo en el aprestamiento para el uso de herramientas y técnicas propias del periodismo, sino bajo la idea de recuperar el espíritu aventurero del reportero que sale al mundo sin quedarse apreciándolo desde la ventana de su navegador de internet.

De nuevo Ramonet (1999) resulta apropiado para ayudar a comprender el fenómeno señalado anteriormente:

La información no tiene valor en sí misma por lo que se refiere, por ejemplo, a la verdad o a su eficacia cívica. La información es, ante todo, una mercancía y, en tanto que tal, está sometida a las leyes del mercado, de la oferta y la demanda, y no a otras leyes como, por ejemplo, los criterios cívicos o éticos.

Por esa razón surge la necesidad no sólo de preguntarse por el perfil de periodista que forma la academia y de lo que esperan los medios de comunicación en ese sentido, porque con ello se estaría descuidando el factor central y 
que hace del periodismo un oficio para el ejercicio de derechos de las comunidades: ¿y cuál es el perfil de periodista que espera la ciudadanía?

Un cuestionamiento inicial sería entonces si, en virtud de lo indicado por Ramonet, la Fundación Universitaria Los Libertadores está formando periodistas de escritorio o periodistas con capacidad para desarrollar trabajo de campo o, en otras palabras, periodismo experiencial. A diario en las radioestaciones pertenecientes a los grandes medios de la prensa industrial de Colombia es frecuente escuchar a periodistas cuyo trabajo consiste en establecer contacto telefónico con personalidades de la vida pública de la nación o en leer reportes de internet, con lo cual nutren las agendas informativas.

Con base en lo anterior, este artículo, derivado de la investigación que responde al título: Estudio sobre competencias requeridas por la industria de los medios para los profesionales egresados de las facultades de comunicación, en contraste con la oferta académica que presentan los distintos programas de comunicación de las universidades de la ciudad de Bogotá, se ocupará de comprender hasta dónde el diseño de los syllabus de las asignaturas del componente disciplinar de periodismo de la Facultad de Ciencias de la Comunicación y Periodismo de la Fundación Universitaria Los Libertadores, guarda coherencia con las necesidades de formación expresadas por un grupo de nueve periodistas con responsabilidades editoriales en igual número de medios de comunicación de la ciudad de Bogotá, que a su vez fueron entrevistados en una etapa anterior de la investigación.

\section{Metodología}

En primera instancia, fueron encuestados nueve periodistas con responsabilidades editoriales de igual número de medios de comunicación con alcance nacional de la ciudad de Bogotá, todos con experiencia en medios de comunicación dada su notable trayectoria para impartir orientación editorial; el sondeo, del mismo modo, incluyó cadenas radiales, canales de televisión, periódicos impresos con presencia en muchas regiones del país y medios digitales, tal y como se lista a continuación: Noticias Uno, Caracol Radio, KienyKe, rcn Radio, Cablenoticias, El Nuevo Siglo, W Radio, Blu Radio y El Espectador.com.

Luego de analizados los resultados de las entrevistas con los periodistas de los medios señalados, fueron revisados quince syllabus correspondientes a las siguientes asignaturas impartidas por el programa de Comunicación social- periodismo de la Fundación Universitaria Los Libertadores: lecturas y lectores, periodismo científico, periodismo digital, periodismo investigativo, periodismo político y económico, periodismo radial, periodismo televisivo, taller de análisis y opinión, taller de crónica y reportaje, taller de informe especial y opinión pública, taller de noticia y entrevista, taller de periodismo ciudadano e investigativo, taller de periodismo narrativo y literatura, taller de radio y taller de televisión.

Una vez seleccionadas las asignaturas, los contenidos consignados en los syllabus fueron contrastados con trece competencias consideradas como necesarias para la formación de un buen periodismo, por las cuales se indagó a los periodistas de medios quienes evaluaron su pertinencia para un óptimo desempeño por parte de los egresados de las facultades de periodismo y comunicación.

En consecuencia, las competencias seleccionadas fueron: buena redacción; destrezas orales; producción multimedia; pensamiento crítico; tecnologías digitales; producción audiovisual; diseño; manejo de tiempo; segunda lengua; trabajo ante las cámaras o micrófonos; trabajo en equipo; cultura general; conocimiento en áreas como economía, política, etc.; conocimiento de eventos de actualidad; técnica de la entrevista; investigación periodística; sentido noticioso.

En un paso posterior se diseñó una matriz en la cual se consignó el contenido referente a cada una de las competencias según los syllabus, teniendo en 
cuenta el total de las quince asignaturas del componente de periodismo del programa. Una vez terminada esta fase se procedió al diseño de una gran matriz en la cual se vaciaron los contenidos de todas las asignaturas, teniendo en cuenta las competencias de formación que fueron identificadas con colores distintivos.

Para facilitar la identificación de las competencias, cada una de ellas fue caracterizada tal y como se indica a continuación:

1. Buena redacción: el estudiante demuestra capacidad de redactar textos periodísticos acorde con las técnicas y propósitos propios de los distintos géneros periodísticos, siguiendo las normas gramaticales y ortográficas de la lengua castellana.

2. Destrezas orales: el estudiante tiene la capacidad de expresar sus pensamientos de forma coherente, así como sostener conversaciones y exponer argumentos mediante el uso de la lengua castellana hablada.

3. Producción multimedia: el estudiante demuestra dominio técnico y conceptual de las herramientas multimediales que le permiten desarrollar contenidos periodísticos a través de la combinación de textos escritos, videos, productos sonoros e imágenes.

4. Pensamiento crítico: el estudiante está en la capacidad de diagnosticar contextos y a partir de su interpretación expone posturas críticas con argumentos sustentables frente a problemas reales, y confronta ideas ajenas con rigor y respeto por las posturas de los demás.

5. Tecnologías digitales: el estudiante opera distintas tecnologías digitales con suficiencia técnica, y comprende su funcionamiento para el servicio de un periodismo de mayor calidad.

6. Producción audiovisual: el estudiante demuestra destrezas en la producción de contenidos audiovisuales mediante el manejo eficaz de las herramientas específicas, y comprende su correcta aplicación para radio y televisión.

7. Diseño: el estudiante es competente en la conceptualización y uso de herramientas propias del diseño para la creación de sitios web, blogs y espacios en la red que permitan compartir contenidos periodísticos.

8. Manejo de tiempo: el estudiante demuestra responsabilidad en cuanto al cumplimiento de sus compromisos laborales en los tiempos pactados, tiene la capacidad para asumir responsabilidades de último momento y para trabajar bajo presión con poca disponibilidad de tiempo; además demuestra eficiencia para organizar logísticamente el tiempo de acuerdo a las tareas que demanda el ejercicio periodístico.

9. Segunda lengua: el estudiante escucha, lee y escribe contenidos periodísticos, así como sostiene conversaciones cotidianas en una segunda lengua, preferiblemente inglés.

10. Trabajo ante las cámaras o micrófonos: el estudiante demuestra suficiencia en la elaboración de argumentos, manejo de tiempos ante situaciones concretas y credibilidad ante las audiencias, cámaras de televisión y micrófonos de radio; asimismo tiene conocimiento técnico de los equipos para la producción de radio y televisión.

11. Trabajo en equipo: el estudiante demuestra capacidad de cooperación con sus compañeros, inteligencia emocional para resolver problemas mediante el diálogo colectivo, buenas relaciones con los demás y tolerancia y respeto ante problemáticas laborales.

12. Cultura general: el estudiante da cuenta de tener conocimientos básicos en áreas de cultura general como historia, arte, derechos humanos, deportes, etc., que es capaz de traducir en contenidos periodísticos y así producir valoraciones argumentales.

13. Conocimiento en áreas como economía, política, etc.: el estudiante demuestra manejo de conceptos básicos de economía, política y 
temas de actualidad en general, y cuenta con la capacidad de traducirlos en contenidos periodísticos.

14. Conocimiento de eventos de actualidad: el estudiante demuestra estar al tanto de eventos de actualidad relacionados con la vida académica, artística, deportiva, entre otros.

15. Técnica de la entrevista: el estudiante demuestra competencias en el dominio de la técnica periodística, y da cuenta de competencias para desarrollar entrevistas en prensa, radio, televisión y medios digitales en general.

16. Investigación: el estudiante demuestra competencias investigativas aplicadas al ejercicio periodístico como manejo de fuentes, búsqueda de datos, diseño de fichas de investigación y análisis de información.

17. Sentido noticioso: el estudiante, además de dominar la técnica para la redacción de noticias, demuestra claridad conceptual y criterio periodístico respecto a cuándo un determinado acontecimiento adquiere la categoría de noticia.

\section{Análisis}

Dicho lo anterior, ahora es necesario pasar a la etapa de contraste entre la matriz que caracteriza a las competencias de formación según lo consignado en los syllabus, en relación con el análisis resultante de las encuestas realizadas en los nueve medios de comunicación en la etapa precedente de la investigación.

Respecto a la competencia denominada buena redacción, el $100 \%$ de los encuestados coincidieron en afirmar su importancia para un desempeño profesional óptimo en los medios de comunicación, competencia que a su vez todos los syllabus de las asignaturas de la universidad mencionan en sus contenidos, aunque no con la profundidad deseada, al menos en algunos casos, pues mientras en la asignatura periodismo científico se la describe con un lacónico «escribir periodismo científico», en periodismo televisivo se le asigna un muy resumido «organización de la redacción y cobertura informativa». Lo anterior supone la existencia de una carencia en cuanto a la definición de la categoría buena redacción como objeto de aprendizaje.

En cuanto a la competencia denominada destrezas orales, el $33.3 \%$ de los encuestados afirmaron que les resulta importante, mientras que el 66.7\% la consideran como muy importante, cifra que asciende a las dos terceras partes. Asimismo, de las quince asignaturas, tres de ellas no enfatizan esta habilidad. Lo anterior permite colegir que para los medios de comunicación una buena expresión oral no es un asunto de segundo orden, lo cual podría suponer que es concordante con la importancia que la universidad le atribuye a esta habilidad, afirmación que resulta por lo menos necesario analizar, puesto que en algunos syllabus el contenido que la describe es idéntico a lo que ocurre con las asignaturas Periodismo Televisivo, Taller de Periodismo Narrativo y Literatura y Redacción para Televisión; lo propio ocurre con Taller de noticia y entrevista y Taller de Crónica y Reportaje; a su vez, el fenómeno se replica en Taller de Informe Especial y Opinión Pública y Taller de Análisis y Opinión.

Si bien el buen redactar y el buen hablar exigen algunas competencias generales que son necesarias independientemente del medio de comunicación, cada medio de comunicación exige del mismo modo ciertas apropiaciones en lo referido a los aspectos escriturales y a la oralidad, especificidades que sería saludable estuvieran incluidas en los syllabus, toda vez que son los syllabus la carta de navegación para los maestros. Manrique (2012), invocando el plan modelo de unEsco, recuerda que éste llama la atención sobre lo que en su concepto debe replantearse en la formación de comunicadores cuando señala que «hay que retomar los ingredientes básicos de la interacción del sujeto con su entorno, es decir, la capacidad de hablar, escuchar, leer y escribir" (p. 115). Esto es, que el meramente redactar según 
los estándares de la gramática de la lengua castellana en este caso, es apenas un atributo accesorio para el periodista si no se le nutre con otras competencias.

Acto seguido, el 77.8\% considera como muy importante la producción multimedia, el 11\% considera que es sólo importante, en tanto que el $11 \%$ restante la define como regular, nivel de importancia que no es concordante con la significación que según los syllabus se le atribuye a esta competencia en la universidad, pues apenas tres asignaturas, equivalentes al $20 \%$, la mencionan de manera expresa. Las asignaturas son periodismo radial, periodismo digital y lecturas y lectores. De lo anterior es posible indicar que la universidad presenta vacíos en cuanto al enfoque de los syllabus para dar respuesta a la necesidad que se desprende de la relevancia que los periodistas de medios le confieren a la capacidad de hacer producciones multimediales. Aquí es necesario acotar que si bien es seguro que muchos de los nuevos periodistas tienen conocimiento del manejo instrumental de las herramientas multimediales, es fundamental una formación para su aplicación conceptual, con sentido desde la práctica profesional periodística.

Insiste Manrique (2012):

[...] todo indica que, una vez más, la profesión periodística y la formación de los periodistas corren a velocidades diferentes. Mientras aquélla avanza rápidamente hacia un escenario de plena digitalización, ésta sigue anclada a menudo en presupuestos teóricos y prácticos que dan la espalda a todos esos câmbios. (p. 115).

Sin duda Manrique acierta cuando indica la prevalencia de lo digital para el ejercicio periodístico moderno, lo cual no está en cuestión, aunque no sobra indicar que, así como la flecha no hace al arquero, los juguetes puestos en su real dimensión jamás harán de un sujeto poco calificado un excelente periodista.
En lo referente a la competencia denominada pensamiento crítico, si bien todos los syllabus revisados la mencionan como objeto de aprendizaje, el $11.1 \%$ de los encuestados la considera como no importante, el $22.2 \%$ como importante y el $66.7 \%$ como muy importante. Asimilar el ejercicio periodístico como el acto mecánico de contar la realidad sin poner en juego un pensamiento crítico, sería tanto como suponer que el periodista no entiende su profesión como un permanente cuestionamiento en relación con el mundo que relata a diario. Que más de la décima parte de un grupo de periodistas entrevistados considere que el pensamiento crítico no es central para el desempeño como periodista por parte de los egresados, es tanto como esperar profesionales que no cuestionen, que simplemente hagan caso, como reza el adagio popular.

Para Caro González y Jiménez Martín (2006), en este aspecto hay un evidente desajuste entre la educación universitaria de periodistas y el mundo profesional que luego se encuentran, porque si bien las universidades se preocupan por la formación crítica de sus egresados, en no pocas ocasiones los medios la desdeñan porque su aspiración gira en torno al aumento de la producción.

Al respecto Caro González y Jiménez Martín (2006) indican atinadamente que:

Las actitudes y habilidades mejor valoradas que perciben los directores y jefes de sección acerca de los periodistas son las relativas al trabajo operativo del mismo, es decir, las destinadas al incremento de la eficacia en las rutinas de producción informativa. (p. 317).

No es un secreto que en Colombia la gran prensa industrial en muchas ocasiones vive en una especie de concubinato con el epicentro de los poderes públicos, económicos y políticos, y que justamente es esa relación la que impide un pensamiento 
deliberante por parte de los profesionales del periodismo.

Para el 22.2\% de los encuestados es importante que los egresados de las facultades de periodismo y comunicación operen tecnologías digitales, mientras que para el $77.8 \%$ es muy importante; igualmente, dicha competencia se explicita en nueve de los syllabus revisados, cifra equivalente al $60 \%$. Aquí es esencial resaltar que en dos de las asignaturas, Taller de Noticia y Entrevista y Taller de Crónica y Reportaje, la alusión a esta habilidad, además de resultar idéntica, es del siguiente tenor: "desarrollar la capacidad de usar la tecnología de la comunicación en la vida académica, laboral y profesional». Si bien lo afirmado resulta una necesidad inapelable para los profesionales modernos, no es menos cierto que el manejo de tecnologías digitales en torno a la práctica periodística tiene unas complejidades que no se resuelven con base en la descripción de generalidades.

\section{Para Ramón Salaverría (2007):}

Todo indica que, una vez más, la profesión periodística y la formación de los periodistas corren a velocidades diferentes. Mientras aquélla avanza rápidamente hacia un escenario de plena digitalización, esta sigue anclada a menudo en presupuestos teóricos y prácticos que dan la espalda a todos esos cambios. Profesión y formación se alejan cada vez más. (p. 1).

La crítica de Salaverría frente a la academia no está fundada en un factor coyuntural como lo es la apropiación de tecnología, sino a un permanente atraso de las escuelas de formación frente a los cambios vertiginosos que experimentan las sociedades modernas, y que por demás sería injusto achacarles por exclusivo a los centros de formación de periodistas.

Por último Salaverría (2007) llama la atención a fin de evitar que las escuelas de formación sigan formando periodistas para el ejercicio de un periodismo con técnicas que ya no tienen lugar en la realidad. Estas confrontaciones en particular son pan de cada día en aquellas universidades donde los maestros tradicionales se encuentran con colegas con nuevas concepciones del oficio, tensiones que antes de avivar la discusión la suprimen en perjuicio de los estudiantes.

Respecto a dichas confrontaciones en las facultades de periodismo, Salaverría (2007), refiriéndose a los maestros y periodistas tradicionales, acota:

[...] suelen ver en el periodismo de los cibermedios un paso atrás en los valores clásicos de la profesión informativa y, por tanto, tienden a menudo a despreciarlos. De hecho con frecuencia no sólo menosprecian el ciberperiodismo, sino incluso a la propia Internet en su totalidad, pues sus herramientas y dinámicas de uso les resultan complejas. (p. 2).

La competencia denominada producción audiovisual se referencia en ocho de las quince asignaturas según los syllabus, cuya apropiación resulta de importancia regular para el $11.1 \%$ de los encuestados, importante a secas para el $66.7 \%$ y muy importante para el $22.2 \%$. Es necesario indicar que sumados los contenidos expresados en los distintos syllabus, la formación en este aspecto podría calificarse como muy completa por la oferta de conocimientos variados y por el soporte conceptual prometido.

Al observar la dinámica de la competencia diseño, esta no es importante para el $22.2 \%$ de los encuestados, es regular para el 33.3\%, importante para el $33.3 \%$ y muy importante para el $11.1 \%$. Tal categoría aparece indicada en apenas tres syllabus correspondientes a las asignaturas Periodismo Digital, Periodismo Radial y Taller de Radio. A la luz de las frías cifras no está demás afirmar que así como para los medios esta competencia es de impacto marginal, lo propio ocurre con su relevancia según los syllabus indicados. 
Los datos anteriores no permiten dilucidar si los editores de medios encuestados entienden en su real dimensión los conceptos de producción audiovisual y diseño, dado que le han atribuido tanta importancia al manejo de tecnologías audiovisuales, pues finalmente tanto aquellas como esta tienden a un mismo propósito: el uso de tecnologías digitales y computarizadas para la difusión de contenidos periodísticos.

Por lo regular los estudiantes de periodismo reciben una formación secuencial según la cual cada uno de los medios de comunicación hace un periodismo en absoluto diferente, visión que supone la idea de que el periodismo es un oficio de corte instrumental, cuyo propósito central consiste en el adiestramiento para el manejo de dispositivos tecnológicos, sin profundizar en los aspectos éticos o conceptuales que suponen su impacto en la profesión.

García Avilés y Martínez Bonastre (2009) al respecto señalan que:

[...] un enfoque transversal se adapta mejor a la concepción del periodismo polivalente como una manera de elaborar información que exige el dominio de múltiples lenguajes, estructuras narrativas adecuadas a los contenidos según el medio, nuevas rutinas productivas y competencias que no pueden limitarse a una única materia sino que han de extenderse al conjunto de asignaturas que conforman el plan de estudios. (p. 243).

Aunque el uso de tecnologías tiene un enfoque específico dependiendo del medio de comunicación en el que tendrán aplicación, su enseñanza no es como un archipiélago con islotes separados los unos de los otros, con saberes fragmentados, o con estudiantes expertos en su apropiación, porque en los medios modernos seguramente ya no es una opción abstraerse del uso de tecnologías, así, y con justa razón se argumente que estas no hacen ni son el periodismo.
Entretanto, la competencia que aparece bajo el nombre manejo del tiempo, resulta importante para el $11.1 \%$ y muy importante para el $88.9 \%$ de los encuestados; esto significa que para el total de los encuestados el manejo de dicha competencia no es un principio secundario, entre otras razones porque en los medios de la gran prensa industrial el periodista trabaja por lo regular bajo márgenes de tiempo reducidos, en atención a la inmediatez, entre otros factores, que dependen de una rigurosa administración del tiempo. De otro lado en el 33.3\% de los syllabus se habla del tiempo como recurso, aunque vale anotar que en uno de ellos, el correspondiente a la asignatura Periodismo Radial, si bien se expone el concepto de instantaneidad, no queda claro como objetivo de aprendizaje.

A propósito del manejo del tiempo, Ramonet (1999) expone:

Desde que, en la segunda mitad del siglo xix, la información experimentó un gran desarrollo, siempre ha existido una relación entre velocidad e información. Ahora, se ha llegado a una situación en que esta relación ha alcanzado un límite tal que plantea problemas, ya que la velocidad es la de la luz y la de la instantaneidad. (p. 2).

En Colombia es frecuente escuchar el término «chiviado", el cual hace referencia a la situación que se presenta cuando un medio de comunicación se adelanta a otro en la emisión de una primicia, lo que implica el descrédito del periodista y del medio que han sido "chiviados» o superados por sus colegas de otros medios. Aquí lo importante no es tanto si la información es de calidad y si tiene el contraste de fuentes adecuado, porque lo relevante es no perder la carrera contra la competencia, olvidando que calidad no es lo mismo que velocidad, frase de perogrullo pero no por ello carente de sentido.

De otro lado, mientras que el manejo de una segunda lengua aparece consignado en dos de los quince 
syllabus, en los que igualmente se transcribe contenido idéntico, esta no es importante para el $11.1 \%$ de los encuestados, es importante para el $22.2 \%$ y muy importante para el 66.7\%. Lo anterior permite inferir que en este aspecto la Fundación Universitaria presenta una carencia que, a juzgar por el resultado de las encuestas a medios, supone una gran dificultad para los egresados del programa de periodismo.

Tal circunstancia pone en debilidad manifiesta a los comunicadores sin manejo de una segunda lengua, puesto que varias de sus interpretaciones en relación con acontecimientos ocurridos en otros centros de poder estarán condicionadas por las traducciones e interpretaciones de periodistas extranjeros. Aun cuando un aporte esencial del periodismo moderno consiste en visibilizar aquello que ocurre en su aldea inmediata, lo local en términos informativos no se basta a sí mismo porque la realidad moderna en su entramado a todo nivel es tan compleja como fragmentada, tan local como global.

Para el $44.4 \%$ de los encuestados es muy importante el trabajo ante las cámaras o micrófonos, porcentaje idéntico de quienes la consideran como una competencia importante, en tanto que para el $11.1 \%$ no es importante. Así mismo esta competencia aparece mencionada en cuatro de los quince syllabus revisados. Ello significa que la universidad presenta lo que podría definirse como una carencia en términos de una formación que dé respuesta al sector productivo. No sobra indicar que, cuando menos, es curioso que dicha competencia no aparezca consignada en el syllabus de la asignatura Taller de Radio, una de las llamadas por su enfoque y objetos de estudio, a enfatizar en el manejo de micrófonos.

Mellado, Simon, Barría y Enríquez (2007) señalan que:

[...] la demanda en la generación de profesionales de la comunicación y su incorporación al mundo laboral debiera ser el reflejo de las relaciones entre academia y su entorno, donde se entrecruzan las decisiones de los entes formadores con las expectativas de los empleadores y de los propios profesionales. (p. 141).

El común de las personas suponen que los periodistas apenas son aquellos que salen por los distintos medios de comunicación, y que entre sus habilidades relevantes deben estar salir por televisión o radio presentando noticias y entrevistando personajes, competencias que apenas cubren la demanda de un sector marginal de las empresas donde podrían desempeñarse los egresados de las facultades de periodismo y comunicación.

Si bien la competencia denominada trabajo en equipo aparece referenciada en siete asignaturas, apenas es mencionada para indicar que los estudiantes desarrollarán trabajos por equipos, sin comprender el trabajo en equipo como un proceso formativo; lo anterior contrasta con los resultados de la encuesta donde se indica que para el $77.8 \%$ de las personas indagadas la competencia en cuestión es muy importante, siendo importante y regular, en igual proporción, en un $11.1 \%$. Hacer trabajo en equipos, como es la tendencia de la universidad, es diferente a conformar equipos, factor que se presenta como una oportunidad para replantear este campo del saber, si de un rediseño de contenidos se tratara.

Caro González y Jiménez Martín (2006), en su investigación denominada Periodistas: el acceso al mercado laboral, la cual fue realizada en España, descubrieron que en más del $70 \%$ de los medios de comunicación de ese país se espera de los periodistas que van a los medios de comunicación que acepten sin discusión las órdenes que se les imparte, conducta que los investigadores atribuyen a estilos de dirección predominantes, autoritarios y con escasa capacidad de orientación al cambio y a la innovación (p. 317). 
Si este fenómeno se replica en Colombia, lo cual no resultaría extraño por las dinámicas propias de los medios que en lo referido al manejo de tiempos dan prioridad a la inmediatez, característica de la gran prensa industrial en el mundo, estaríamos ante un doble fenómeno que a su vez estaría dificultando el trabajo en equipo: estudiantes sin la suficiente preparación para sumarse a equipos de trabajo, donde son aceptados como un elemento externo al que simplemente es necesario incorporar y no como factor humano indispensable, que además se enfrentan a estructuras de mando horizontales donde lo importante es quién manda y no a quiénes y cómo manda.

Cultura General aparece en dos asignaturas, Periodismo Científico y Taller de Radio, competencia que, asimismo, resulta muy importante para el $88.9 \%$ e importante para el $11.1 \%$ de los encuestados. Este evidente contraste entre lo que ofrece la universidad y lo que esperan los empresarios de los medios de comunicación de la prensa industrial puede ser atenuado debido a que los conocimientos que los estudiantes cosechan en todas las asignaturas son justamente eso, cultura general, que se suman a todo tipo de experiencias personales.

En su obra Los Cínicos no Sirven para este Oficio el maestro Ryszard Kapuscinski (1980), tal vez uno de los periodistas más importantes del siglo $\mathrm{xx}$, al respecto acota:

Hay profesiones para las que, normalmente, se va a la universidad, se obtiene un diploma y ahí se acaba el estudio. Durante el resto de la vida se debe, simplemente, administrar lo que se ha aprendido. En el periodismo, en cambio, la actualización y el estudio constantes son la conditio sine qua non. (p. 17).

Las sociedades modernas, a diferencia de aquellas que le precedieron, sufren permanentes cambios en todos los ámbitos de la vida pública, y por tan- to es responsabilidad del periodista, además de comprender las complejidades que lo circundan, anticiparse a ellas si es posible, lo cual no logrará sin ser un intelectual y sin una cultura general, sin tener abundante información y la capacidad suficiente para comprenderla.

Conocimientos en áreas como política, economía, etc., como competencia de conocimiento, está indicada en tres syllabus, correspondientes a las asignaturas Taller de periodismo ciudadano e investigativo, Periodismo Político y Económico y Periodismo Investigativo, competencia que, entretanto, es muy importante para el 55.6\% de los entrevistados e importante para el 33.3\%. Aquí, del mismo modo, es necesario aclarar que si bien el impacto explícito de la competencia referenciada es marginal en los syllabus, es muy seguro que muchas otras asignaturas estén atravesadas por estos saberes específicos.

Conocimiento de eventos de actualidad aparece mencionada en el syllabus de periodismo científico con un lacónico: «Hace monitoreo a noticias científicas y medios especializados», factor que a su vez es reconocido como regular por el $11.1 \%$ de los encuestados, e importante y muy importante para el $44.4 \%$ de los encuestados, respectivamente. En buena medida, el periodismo moderno nutre sus contenidos gracias a los llamados eventos de actualidad y cuyo conocimiento es fundamental, a no ser que se trate del llamado periodismo en frío que depende de otros factores.

Una vez más, Manrique (2012), en relación con lo anterior, retoma El Plan Modelo de Unesco, el cual hace referencia a la formación de periodistas; y que en uno de los apartados expone que: «Se enfoca en que "el estudiante tenga un conocimiento general, es decir, que no tenga sólo destrezas periodísticas sino también una sólida cultura general y una educación interdisciplinaria”» (p. 114). Es decir, a la sociedad en su conjunto de muy poco o nada le servirá un comunicador experto en el manejo de herramientas tecnológicas, las cuales, aunque son 
necesarias para ciertos aspectos instrumentales de la información, no pueden obviar el hecho de que la fortaleza del comunicador estriba en su potencia como transformador de cultura y agente crítico de su tiempo.

Si se trata de ir más allá, incluso el conocimiento, por parte del comunicador, de eventos de actualidad o de asuntos de economía y política, resultan casi que accesorios si su fortaleza consiste en reproducirlos o en no ayudar a su comprensión. Surge entonces la pregunta sobre si el periodista de calidad es un replicador de aquello que pasa por la lente de sus sentidos o si, por el contrario, se constituye en un testigo activo con capacidad para comprender, analizar y facilitarles a los usuarios de sus contenidos tales atributos.

Técnica de la entrevista aparece explicitada en cuatro syllabus, aunque en el correspondiente a la asignatura Periodismo Radial no señala cómo se convertirá en un objeto de aprendizaje. Por su parte, el $66.6 \%$ de los encuestados consideran muy importante saber hacer entrevistas, importante el $22.2 \%$ y regular el $11.1 \%$. Esta falencia, que según los syllabus presenta la universidad, se agudiza en el ejercicio profesional, toda vez que un factor central para producir buen periodismo está soportado en entrevistas de calidad.

Pese a que la entrevista como género periodístico se vale de algunas técnicas para alcanzar su mayor eficacia, técnicas que el comunicador está en la responsabilidad de conocer, es necesario aclarar que una cosa es la técnica y otra muy distinta comprender a plenitud sus propósitos. Santiago Tejedor (2007) expone lo siguiente, sobre la formación de comunicadores:

El conocimiento instrumental o técnico es importante en la formación de los futuros profesionales del periodismo online. Sin embargo, ni es el más importante ni el único. No obstante, la reflexión en torno a las competencias que ha de poseer el ciberperiodista ha desembocado en muchos casos en una conclusión errónea: el ciberperiodista ha de ser un experto en el uso de las nuevas herramientas técnicas. (p. 28).

Investigación periodística aparece indicada en once syllabus, aunque los contenidos propuestos son idénticos para Taller de informe especial y opinión pública y para Taller de análisis y opinión. Igualmente, la investigación periodística es importante para el 33.3\% y muy importante para el $66.6 \%$ de los interrogados. Un aspecto destacable y que se deriva de la revisión de los syllabus tiene que ver con que la investigación periodística aquí trasciende la idea de que se investiga en periodismo para destapar escándalos, labor que no es desdeñable, pero que está lejos de definir la investigación periodística como su único propósito.

Sería saludable partir del principio de que todo contenido periodístico debería estar soportado por un mínimo acervo investigativo, sano para la sociedad y ético para el periodista, tanto por rigor como para superar la idea de que el periodismo investigativo consiste en mostrar las desgracias de la sociedad o metérsele debajo de las cobijas a las llamadas celebridades.

Del mismo modo, el periodismo investigativo pareciera estar relegado a la tarea exclusiva de sondear portales de internet para luego replicar sus contenidos. Al respecto, retomamos a Salaverría (2007) cuando indica:

[...] qué le ocurriría a un periodista de cualesquiera de estos medios clásicos si hoy se le privara de internet como recurso documental y de investigación. Probablemente sería incapaz de realizar su trabajo. El periodismo del siglo xxi, cualquiera que sea su modalidad, ya no se puede concebir al margen de internet. (p. 1). 
Salaverría (2007) parte de dos presupuestos: que no es posible el periodismo del siglo xxi sin internet, y que los periodistas de la llamada vieja guardia no podrían hacer su trabajo sin el aporte de las herramientas digitales. Seguramente una computadora y una buena conexión a internet son esenciales para cualquier periodista, pero una excelente crónica o un notable reportaje jamás se escribirían por sí solos de manera superior en una máquina de escribir que en una computadora, por potente que ésta sea, reflexión que necesariamente sería pertinente para las escuelas de periodismo.

El 100\% de los encuestados coincide en señalar que el sentido noticioso es una competencia muy importante para la práctica profesional periodística, la cual aparece reseñada en cuatro de los quince syllabus referenciados. Esta competencia de formación está consignada en las asignaturas Taller de noticia y entrevista, como es natural, Periodismo político y económico y Periodismo investigativo y Periodismo científico. Podría afirmarse que lo anterior no significa la presencia de una falencia insuperable por parte de la universidad, toda vez que, por un lado, todas las asignaturas de periodismo no necesariamente tienen la responsabilidad de enfatizar en los aspectos noticiosos, y porque el periodista moderno es mucho más que un simple redactor de acontecimientos de último momento.

Si reportar los acontecimientos a la mayor velocidad posible fuera la definición de noticia como género periodístico, entonces traer a Ramonet (1999) resulta pertinente cuando él indica que: «La actualidad es básicamente lo que dice el medio de comunicación dominante. Si éste afirma que algo forma parte de la actualidad, los demás medios de comunicación lo repetirán» (p. 3). Con base en lo señalado por Ramonet, en muchas ocasiones lo noticioso obedece, no tanto a la importancia del acontecimiento reportado en términos de su impacto frente a la sociedad y su valor por ser de interés general, sino a la prevalencia caprichosa que el medio de comunicación le atribuye; por consiguiente, la noticia en algunas ocasiones termina siendo no aquello que representa el interés colectivo, sino la espuma que circula a través de los distintos medios de información masivos.

\section{Conclusiones}

En muchos de los syllabus aparecen las competencias de formación apenas mencionadas, lo cual indica que desde la planeación curricular no se está complejizando el conocimiento. Tal situación no sólo se explica por el hecho de que se trate de frases en extremo resumidas, sino porque tampoco muestran un horizonte que permita enfocar conceptualmente la dinámica de las competencias a depurar.

Otro elemento a destacar tiene que ver con que la universidad, al menos en lo que refleja el diseño curricular, presenta debilidades en los aspectos de formación que tienen que ver con la apropiación de recursos tecnológicos para la producción de contenidos periodísticos. Aun cuando no está en discusión que al periodista no lo hacen los llamados juguetes o el manejo de tecnologías, la dinámica de los medios actuales exige competencias en el dominio de tecnologías y en el diseño de contenidos que resulten de mayor atractivo para los usuarios de medios.

En lo tocante a lo anterior, Tejedor Calvo (2008) acota:

El ciberperiodismo exige cambios que afectan a las características de los mensajes informativos —adaptados a las posibilidades del medio online-, a las etapas del proceso productivo -en las que se han de aplicar nuevas rutinas de producción- y a la organización interna de los medios digitales - dotados de nuevas estructuras y flujos de producción. (p. 1). 
Asimismo, Tejedor Calvo (2007) insiste en que:

Estas demandas de carácter formativo plantean un reto cuya consecución se enmarca en el ámbito académico: la revolución del ciberperiodismo exige también una serie de transformaciones en el planteamiento de los estudios de Periodismo. La inclusión curricular del ciberperiodismo es, hoy por hoy, un hito tan necesario como inalcanzado. (p. 1).

Un hecho que llama la atención tiene que ver con la importancia que los periodistas encuestados le atribuyen a la competencia pensamiento crítico, pues si bien el $66.7 \%$ la mayoría le atribuyen la categoría de muy importante para el ejercicio del periodismo, no deja de ser curioso que el $30.3 \%$ restante no la eleve a dicha categoría, lo cual contrasta con la propuesta de la universidad, dado que los quince syllabus la mencionan de una u otra manera.

Dos cuestionamientos surgen al respecto: por un lado surge la interrogante sobre si la academia está más dispuesta que los mismos medios de comunicación a expresar una postura crítica respecto de la realidad, y, por otro lado, aparece la pregunta sobre qué tan incómodos pueden llegar a ser para la gran prensa industrial los periodistas formados en la cultura de la deliberación y el disenso.

Kapuscinski (1980) pone la flecha en el centro de la discusión cuando afirma:

En efecto, la mayoría de los directores y de los presidentes de las grandes cabeceras y de los grandes grupos de comunicación no son, en modo alguno, periodistas. Son grandes ejecutivos.Seguramente los empresarios dueños de empresas de comunicación ven a sus organizaciones de medios como un negocio, y toda postura deliberante que atente contra sus propósitos comerciales surge como una rebeldía que es necesario acallar, y si en el centro de los intereses en juego está un periodista de a pie o un jefe de redacción, no será cuestión de artes adivinatorias suponer el desenlace final de la controversia. (p. 34).

Podría pensarse que, al menos en una democracia sana, es esperable que todos los periodistas expresen un pensamiento crítico, y que además ese pensamiento fuera valorado sin cortapisas por jefes de redacción, directores de medios y, en general, por toda la cadena de mando de los medios industriales.

En consecuencia, una pregunta de fondo sería: ¿qué esperan los jefes de redacción de un periodista cuando no le atribuyen a su capacidad de pensar con criterio máxima valoración? Seguramente la respuesta está dada, en parte, por la valoración que esos mismos jefes de redacción le atribuyen a la competencia noticiosa, a la que en su totalidad califican como muy importante; es decir, necesitan redactores de noticias, que también deben ser construidas tan rápido como sea posible, o al menos eso se desprende de la calificación de muy importante que el $88.9 \%$ de esos mismos redactores le atribuye a la competencia manejo del tiempo.

Delgado Socatellyi (2001) presenta desde el deporte una propuesta que es aplicable a otros enfoques de la formación de periodistas:

El periodismo deportivo no debe perder nunca de vista los objetivos de la educación física, el deporte, la recreación y la salud, por cuanto son la base que orienta la información deportiva. El profesional debe prepararse para encauzar la comunicación hacia la salud, la dirección de los procesos de desarrollo personal, la formación del carácter, el fortalecimiento del espíritu familiar, de los valores cívicos y uso de su tiempo libre. (p. 101). 
Así mismo, las escuelas de periodismo tienen en el horizonte del ejercicio de derechos de todos los ciudadanos el fortalecimiento de la democracia, la vida en solidaridad y la vigilancia permanente de todos los centros de poder, el enfoque perfecto para la formación de profesionales de la comunicación, independientemente de si la decisión es hacer periodismo tradicional o de avanzada.

Entretanto qué ocurre con quienes se forman en las escuelas de periodismo y sus expectativas no pasan por convertirse en periodistas de los grandes medios, porque sus intereses giran en torno a otros propósitos profesionales: ¿está la academia preparada para atender esas otras demandas? Mellado, Simon, Barría y Enríquez (2007):

En este contexto y al menos desde la perspectiva latinoamericana, hoy se refuta una creencia albergada y defendida por muchos pero muy poco trabajada tanto por la academia como por los propios profesionales: suponer que el profesional que no trabaja en medios de comunicación de masas, no es periodista. (p. 146).

Surge entonces una oportunidad que al menos de parte de la Fundación Universitaria los Libertadores no está plasmada de manera concreta en los syllabus analizados, y que a su vez se erige como una posibilidad de acción, consistente en pensar el enfoque de formación no como una respuesta a los medios de la prensa industrial, sino como una oportunidad para enfatizar en la formación de comunicadores con la capacidad y el criterio para involucrarse con procesos sociales de base en el periodismo comunitario y alternativo, en la formación de ciudadanos con competencias comunicativas para el ejercicio pleno de derechos, y en la investigación para el diagnóstico y la solución de problemas comunitarios desde la investigación aplicada en comunicación.

\section{Referencias}

Arroyave, J., y Blanco, I. (2005). Cómo perciben los periodistas su profesión: entre el agotamiento y la fascinación. Investigación y desarrollo, 13 (2), 364-389.

Cabalin, C., y Lagos, C. (2012). Enseñanza del periodismo en Chile y globalización. Revista Signo y Pensamiento, XXXI (61), 158-170.

Caro, F., y Jiménez, G. (2006). Periodistas: el acceso al mercado laboral. Revista Ámbitos (15), 313-234.

Chacón, J., y Cuesta, O. (2016). La radio alternativa y comunitaria en Bogotá, escenario de convergencia periodística en una complejidad cultural. Memorias. XIII Congreso Latinoamericano de Investigadores de la Comunicación (pp. 13-22). Ciudad de México: Universidad Autónoma Metropolitana.

Cuesta, O. (2012). Apuntes sobre el papel del comunicador, la comunicación alternativa y la cultura. En O. Cuesta, Yo informo, tú opinas, ellos se forman, todos comunicamos. Bogotá: Fundación Universitaria Los Libertadores.

De Mendonça, T., Pereira, F., y Leal-Adghirni, Z. (2012). Formación y producción periodística en Internet: desafíos y perspectivas en el escenario brasileño. Palabra Clave, 15( 1), 26-53.

De Oliveira, F. (2011). Teoría del periodismo. Sevilla: Comunicacion Social Ediciones y Publicaciones.

Delgado, G. (2001). Guía de orientación para el diseño de una propuesta curricular para la formación profesional del periodista deportivo en Costa Rica. Revista de Ciencias Sociales, IV (94), 98-112. 
García, J., y Martínez, O. (2009). Competencias en la formación universitaria de periodistas a través de nuevas tecnologías. Revista de la Facultad de Ciencias Sociales y Jurídicas de Elche, 1 (4), 239-250.

Grassler, M. (2013). Educar en periodismo de datos. Estudio de casos y propuestas de inclusión curricular. [Tesis de maestría]. Universidad Autónoma de Barcelona, Barcelona.

Kapuscinski, R. (17 de junio de 1980). Los cínicos no sirven para este oficio. Barcelona: Anagrama. Recuperado de https:// koralieucm.files.wordpress.com/2010/09/ kapuscinski-los-cinicos-no-sirven-para-esteoficio.pdf

Lemos, L. E. (1993). Chile: Educación superior en periodismo y comunicación. Chasqui. Revista Latinoamericana de Comunicación(44), 59-60.

Manrique, J. (2012). Enseñar periodismo para leer y narrar la sociedad del siglo XXI. Cuadernos de Información, 30, 111-122.

Mellado, C., Simon, J., Barría, S., y Enríquez, J. (2007). Investigación de perfiles profesionales en periodismo y comunicación para una actualización curricular permanente. Revista Zer, 139-164.
Narváez, A. (2013). Educación y comunicación, del capitalismo informacional al capitalismo cultural. Bogotá: Universidad Pedagógica Nacional.

Ramonet, I. (1999). El periodismo del nuevo siglo. Revista La Factoría (8).

Romero-Domínguez, L. (2006). Cómo formar a los informadores de la era digital: el periodista integrado o el «malabarista» de la comunicación. Análisis y propuestas en torno al periodismo digital. VII Congreso Nacional Periodismo Digital Universidad de Sevilla, Sevilla.

Salaverría, R. (2007). Formar periodistas en la era de Internet. Recuperado de : http://dadun. unav.edu/bitstream/10171/7379/1/formar periodistas_en_la_era_de_internet0001.pdf

Tejedor Calvo, S. (2007). Ciberperiodismo y universidad: la inclusión curricular del periodismo on-line. Recuperado de http:// fama2.us.es/fco/digicomu/37_24.pdf

Tejedor Calvo, S. (2008). Ciberperiodismo y universidad: diagnósticos y retos de la enseñanza del periodismo on-line. Anàlisi, 36, 25-39. 